\title{
Differences between Atrial Fibrillation Detected before and after Stroke and TIA: A Systematic Review and Meta-Analysis
}

\author{
Sebastian Fridman ${ }^{a} \quad$ Amado Jimenez-Ruiz ${ }^{b}$ Juan Camilo Vargas-Gonzalez ${ }^{\text {b, c }}$ \\ Luciano A. Sposato ${ }^{a, b, c, d, e, f}$ \\ aDepartment of Clinical Neurological Sciences, Schulich School of Medicine and Dentistry, Western University, \\ London, ON, Canada; ${ }^{b}$ Heart \& Brain Laboratory, Western University, London, Canada, Western University, London, \\ ON, Canada; 'Department of Epidemiology and Biostatistics, Schulich School of Medicine and Dentistry, London, \\ ON, Canada; 'Department of Anatomy and Cell Biology, Schulich School of Medicine and Dentistry, London, ON, \\ Canada; ${ }^{e}$ Robarts Research Institute, London, ON, Canada; ${ }^{f}$ Lawson Health Research Institute, London, ON, Canada
}

\section{Keywords}

Stroke · Atrial fibrillation · Prevention · Recurrence ·

Transient ischemic attack

\section{Abstract}

Background: Preliminary evidence suggests that patients with atrial fibrillation (AF) detected after stroke (AFDAS) may have a lower prevalence of cardiovascular comorbidities and lower risk of stroke recurrence than AF known before stroke (KAF). Objective: We performed a systematic search and meta-analysis to compare the characteristics of AFDAS and KAF. Methods: We searched PubMed, Scopus, and EMBASE for articles reporting differences between AFDAS and KAF until June 30, 2021. We performed random- or fixed-effects metaanalyses to evaluate differences between AFDAS and KAF in demographic factors, vascular risk factors, prevalent vascular comorbidities, structural heart disease, stroke severity, insular cortex involvement, stroke recurrence, and death. $\boldsymbol{R} \boldsymbol{e}$ sults: In 21 studies including 22,566 patients with ischemic stroke or transient ischemic attack, the prevalence of coronary artery disease, congestive heart failure, prior myocardial infarction, and a history of cerebrovascular events was significantly lower in AFDAS than KAF. Left atrial size was smaller, and left ventricular ejection fraction was higher in AFDAS than KAF. The risk of recurrent stroke was $26 \%$ lower in AFDAS than in KAF. There were no differences in age, sex, stroke severity, or death rates between AFDAS and KAF. There were not enough studies to report differences in insular cortex involvement between AF types. Conclusions: We found significant differences in the prevalence of vascular comorbidities, structural heart disease, and stroke recurrence rates between AFDAS and KAF, suggesting that they constitute different clinical entities within the AF spectrum. PROSPERO registration number is CRD42020202622.

(c) 2021 The Author(s).

Published by S. Karger AG, Basel

\section{Introduction}

Evidence from clinical and experimental research suggests that atrial fibrillation (AF) detected after stroke (AFDAS) is different from known AF (KAF) before stroke occurrence $[1,2]$. It has been hypothesized that detection bias resulting from prolonged cardiac monitoring after stroke may lead to the detection of low-risk AFDAS that may not always have a causal role [1]. Also, neurogenic karger@karger.com www.karger.com/ced

Karger $\frac{1}{\%}$
(C) 2021 The Author(s)

Published by S. Karger AG, Basel

This is an Open Access article licensed under the Creative Common Attribution-NonCommercial-4.0 International License (CC BY-NC) (http://www.karger.com/Services/OpenAccessLicense), applicable to the online version of the article only. Usage and distribution for commercial purposes requires written permission.
Correspondence to:

Luciano A. Sposato, luciano.sposato@lhsc.on.ca 
mechanisms in stroke patients with relatively healthy hearts may trigger transient paroxysms of lower-burden AF. Identifying differences between AFDAS and KAF is important for characterizing the risk of stroke recurrence and potential differences in the risk/benefit ratio of oral anticoagulation. In line with this hypothesis, in a population-based study, AFDAS did not entail a higher risk of stroke recurrence than no AF in fully adjusted models. In contrast, KAF was associated with $25 \%$ increased risk [3]. Based on this evidence, we hypothesized that AFDAS has a lower prevalence of cardiovascular comorbidities and structural heart disease and a lower risk of stroke recurrence than KAF [4]. We performed a systematic search and meta-analysis to compare the characteristics and prognosis of AFDAS and KAF.

\section{Materials and Methods}

The study protocol was developed based on the 2020 Preferred Reporting Items for Systematic Reviews and Meta-Analyses Protocols guidelines [5] and is published elsewhere [4]. We performed a systematic search of articles published until June 30, 2021, including patients with ischemic stroke or transient ischemic attack (TIA) reporting differences between AFDAS and KAF. We searched PubMed, Scopus, and EMBASE according to prespecified search terms (online suppl. Tables S1-S3; for all online suppl. material, see www.karger.com/doi/10.1159/000520101) and study protocol [4]. We also included all titles listed in the PubMed functions "Similar articles" and "Cited by" (online suppl. Tables S4 and S5). Two authors screened all titles (A.J-.R. and L.A.S.) and handled differences by consensus. To be included, studies had to comprise patients with a diagnosis of ischemic stroke or TIA reporting any of the following data: demographics (age and sex), risk factors (hypertension, diabetes mellitus, dyslipidemia, and $\mathrm{CHA}_{2} \mathrm{DS}_{2}$ VASc score), vascular comorbidities (coronary artery disease, congestive heart failure, prior myocardial infarction, peripheral artery disease, previous stroke, or TIA), chronic kidney disease, cardiac structural characteristics (left atrial diameter and left ventricular ejection fraction), stroke severity (National Institutes of Health Stroke Scale), insular cortex involvement, and outcomes (stroke recurrence and death) in groups with AFDAS and KAF. We excluded review articles, letters to the editor, editorial articles, conference papers with incomplete information, studies with small sample sizes ( $<30$ subjects), and publications with duplicated cohorts or without information an any of the variables specified in the inclusion criteria. Data extraction was performed by a single author (L.A.S.), subsequently audited by a second author (S.F.) and additionally reviewed twice by a single author (L.A.S.). We initially planned to perform fixed or random effects meta-analyses depending on heterogeneity (random-effects analyses when $I^{2}$ was $>40 \%)[4,6]$. However, we found that study populations, diagnostic methods, and the setting of the clinical diagnosis of AFDAS were substantially diverse, suggesting that heterogeneity was present in all analyses despite low $I^{2}$ statistics. As such, we performed random-effect models for all variables to obtain more reliable and conservative estimates. Additionally, we performed fixed-effect analyses for variables with $I^{2}<40 \%$ to identify potential inconsistencies. Performing random-effect models for all variables was also considered as a way to avoid assigning a disproportionate weight to a few larger studies, since random-effect analyses give relatively more weight to smaller studies than fixed-effects approaches [6]. We estimated odds ratios (OR) and 95\% confidence intervals (95\% CI) for dichotomic variables and standardized mean differences (SMD) for continuous variables. We stratified the magnitude of SMD (Cohen's $d$ ) into small $(d=0.2)$, medium $(d=0.5)$, and large $(d=0.8)$ [7]. As prespecified in the study protocol, we applied the "leave-one-out" procedure, influence analyses, and graphical display of study heterogeneity (GOSH) plots to account for sources of heterogeneity for variables with $\geq 10$ studies and high heterogeneity $\left(I^{2}>40 \%\right)[4]$. As sensitivity analyses, we generated additional estimates after excluding studies identified as outliers and causing significant heterogeneity. We evaluated differences between AFDAS and KAF in variables comprised in the inclusion criteria. We used funnel plots to assess publication bias. We applied the Critical Appraisal Checklist for cohort, case-control, and observational studies to evaluate the risk of bias [8]. We used this tool instead of the one originally stated in the study protocol because it has a greater versatility for assessing different types of studies. The risk of bias was assessed by a single author (J.C.V.-G.) and audited by a second author (L.A.S.). We did not perform meta-analyses for variables reported in $<3$ studies. We performed all analyses with $\mathrm{R}$ V.3.6.2 ("Meta" and "Metaphor" packages). Most of the data used in this study are available in the online supplementary file. Full datasets will be made available upon reasonable request.

\section{Results}

After screening 33,795 titles, we included 21 studies comprising 22,566 patients (online suppl. Fig. S1). Hypertension (OR 0.79, 95\% CI: 0.68, 0.92; 16 studies; $I^{2}=$ $34 \%$ ), dyslipidemia (OR 0.82, 95\% CI: 0.73, 0.92; 11 studies; $\left.I^{2}=0 \%\right)$, coronary artery disease (OR $0.50,95 \% \mathrm{CI}$ : $0.42,0.61 ; 8$ studies; $I^{2}=48 \%$ ), prior myocardial infarction (OR 0.60, 95\% CI: 0.46, 0.79; 5 studies; $I^{2}=7 \%$ ), congestive heart failure (OR 0.37, 95\% CI: $0.31,0.44 ; 12$ studies; $I^{2}=17 \%$ ), peripheral artery disease (OR $0.44,95 \%$ CI: $0.29,0.68 ; 7$ studies; $I^{2}=68 \%$ ), previous stroke (OR 0.38, 95\% CI: $0.25,0.58 ; 5$ studies; $I^{2}=43 \%$ ), and previous stroke or TIA (OR 0.57, 95\% CI: 0.42, 0.77; 4 studies; $I^{2}$ $=0 \%)$ were significantly less prevalent in AFDAS than in KAF (Fig. 1a; online suppl. Fig. S2, S3, S5-S10). The $\mathrm{CHA}_{2} \mathrm{DS}_{2}$-VASc score was $50 \%$ lower in patients with AFDAS than in those with KAF (SMD -0.47 95\% CI: $-0.60,-0.34$; 9 studies, $I^{2}=0 \%$ ) (Fig. $1 \mathrm{~b}$; online suppl. Fig. S4). Mean left atrial diameter was smaller (SMD $-0.65,95 \%$ CI: -0.99 to $-0.31 ; 5$ studies; $\left.I^{2}=41 \%\right)$ and the left ventricular ejection fraction was larger (SMD 0.25, 95\% CI: $0.20-0.30$; 5 studies; $I^{2}=0 \%$ ) in AFDAS 
a

\begin{tabular}{|c|c|c|c|c|c|}
\hline Outcome & $\begin{array}{l}\text { No. of } \\
\text { studies }\end{array}$ & $\begin{array}{l}\text { No. of } \\
\text { patients }\end{array}$ & $\begin{array}{r}\text { Lower risk } \\
\text { in AFDAS } \\
\text { than KAF }\end{array}$ & $\begin{array}{l}\text { Higher risk } \\
\text { in AFDAS } \\
\text { than KAF }\end{array}$ & $\begin{array}{l}\text { Odds ratio } \\
(95 \% \mathrm{Cl})\end{array}$ \\
\hline
\end{tabular}

Demographics

Female Sex

$17 \quad 17,495$

Risk Factors

Hypertension

Diabetes

Dyslipidemia

$16 \quad 17,489$

$16 \quad 17,487$

$11 \quad 15,531$

Comorbidities

Coronary artery disease

Prior myocardial infarction

Congestive heart failure

Peripheral artery disease

Prior stroke

Prior stroke or TIA

Chronic kidney disease

15,531

Outcomes

Recurrent stroke

Death

$\begin{array}{rr}8 & 15,395 \\ 5 & 8,854 \\ 12 & 16,350 \\ 7 & 8,440 \\ 5 & 8,935 \\ 4 & 2,293 \\ 3 & 1,603\end{array}$

$0.98(0.76,1.25)$

$0.79(0.68,0.92)$

$0.85(0.70,1.03)$

$0.82(0.73,0.92)$

$0.50(0.42,0.61)$

$0.60(0.46,0.79)$

$0.37(0.31,0.44)$

$0.44(0.29,0.68)$

$0.38(0.25,0.58)$

$0.57(0.42,0.77)$

$0.52(0.18,1.51)$

, 603

$\mapsto$

$\longmapsto$

$0.74(0.58,0.95)$

$0.87(0.56,1.37)$

$6 \quad 6,427$

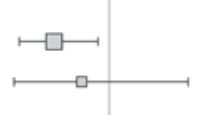

$\begin{array}{lllll}0.0 & 0.5 & 1.0 & 1.5 & 2.0\end{array}$

b

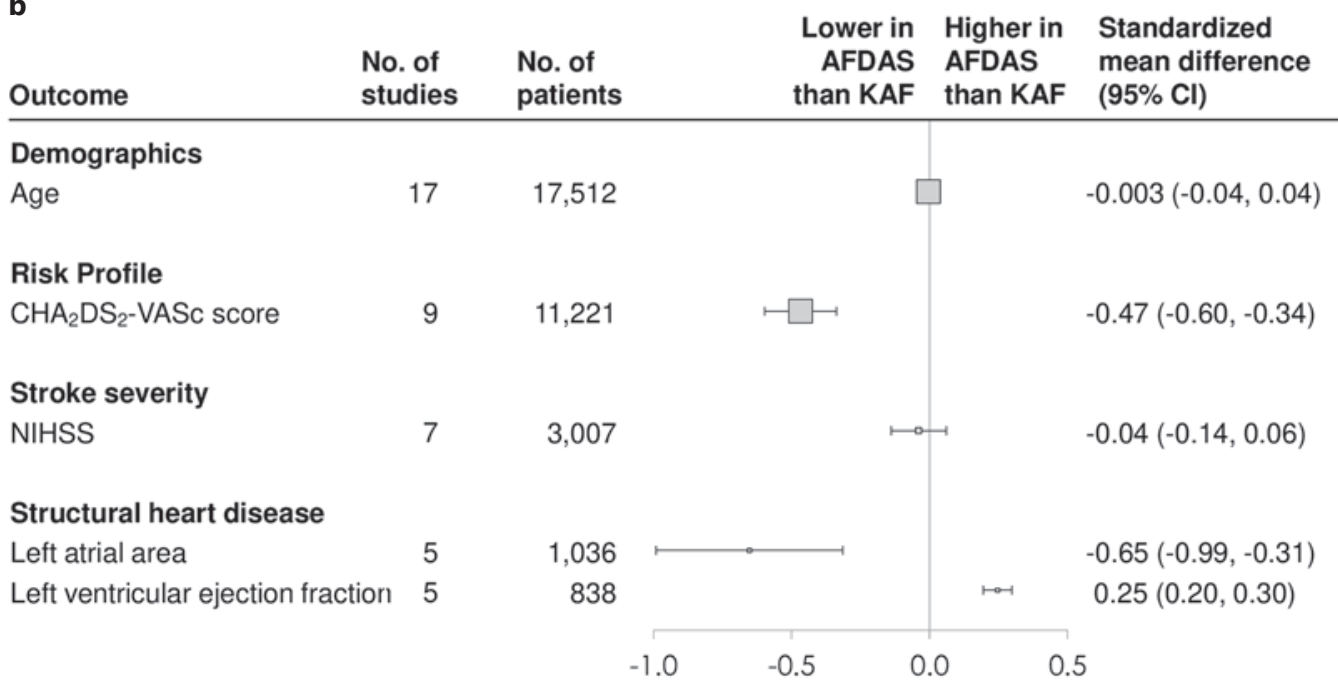

Fig. 1. Summary measures of all meta-analyses comparing AFDAS and KAF. AFDAS, atrial fibrillation detected after stroke; KAF, known atrial fibrillation; NIHSS, National Institutes of Health Stroke Scale; TIA, transient ischemic attack. 
Table 1. Main characteristics of included studies $[3,9,10,14-31]$

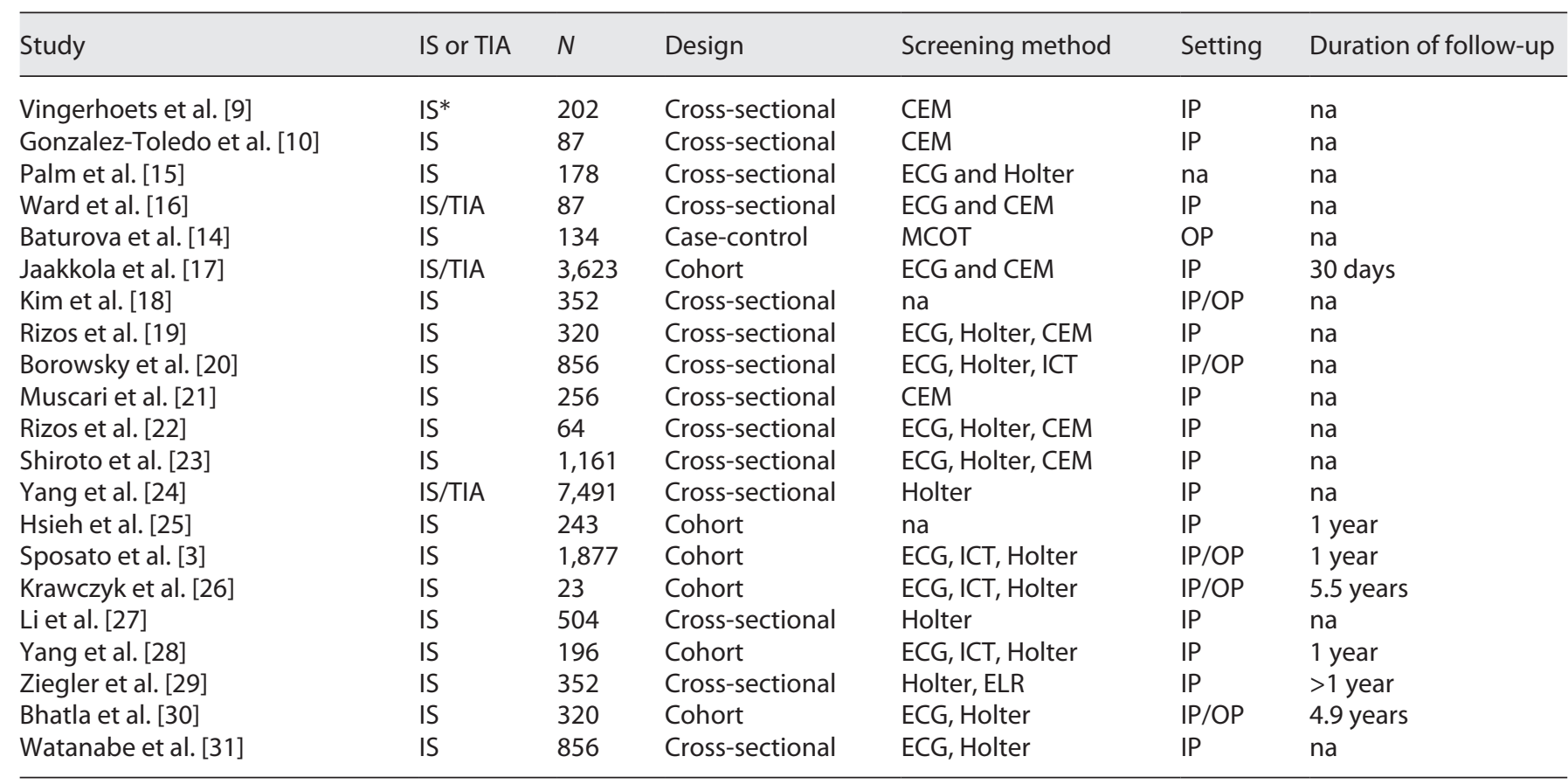

Online supplementary Table S6 (online suppl. material) summarizes the demographic characteristics and baseline risk factors of patients included in all studies. CEM, continuous inpatient electrocardiographic monitoring; ECG, admission or inhospital electrocardiogram; ELR, external loop recorder; ICT, inhospital cardiac telemetry; IP, inpatient; IS, ischemic stroke; MCOT, mobile cardiac outpatient telemetry; OP, outpatient; TIA, transient ischemic attack. * Ten patients had an intracerebral hemorrhage.

than in KAF patients (Fig. 1b; online suppl. Fig. S11 and S12). AFDAS was associated with a $26 \%$ lower risk of stroke recurrence than KAF (OR 0.74, 95\% CI: 0.58-0.95; 3 studies; $I^{2}=0 \%$ ) (Fig. 1a; online suppl. Fig. S12). The absolute risk difference between AFDAS and KAF was $-2.6 \%$ (95\% CI: -3.7 to $-1.6 \%)$. There were no differences between AFDAS and KAF in the remaining prespecified variables (online suppl. Fig. S13-S19). We were unable to perform a meta-analysis for insular cortex involvement because we only identified 2 publications reporting differences between AFDAS and $\operatorname{KAF}[9,10]$. The risk of bias was overall high. Among 6 cohort studies, there was one in which the risk of bias was high because no strategies for addressing confounding were described (online suppl. Fig. S20). We included only 1 case-control study, which showed only moderate concern of risk of bias (online suppl. Fig. S21). Among 14 cross-sectional studies, 11 evidenced at least 1 criterion for risk of bias (online suppl. Fig. S22). Fixed-effect models applied to variables with $I^{2}>40 \%$ showed no differences relative to random-effect models (online suppl. material).

Atrial Fibrillation Detected after Stroke

\section{Discussion}

We found that the prevalence of vascular risk factors, the $\mathrm{CHA}_{2} \mathrm{DS}_{2}$-VASc score, and cardiovascular comorbidities were consistently and significantly lower in AFDAS than in KAF. Patients with AFDAS also had a smaller left atrial diameter and higher left ventricular ejection fraction. These findings suggest that patients with AFDAS may have a relatively more benign vascular profile than those with KAF, explaining the lower risk of stroke recurrence. The healthier vascular profile and less severe structural heart disease may explain the reported lower burden of AF in AFDAS patients [11], which in turn may further contribute to the lower risk of stroke recurrence. These findings are mostly based on pooled data from retrospective observational studies and thus require further confirmation in prospective studies comparing AFDAS and KAF characteristics and outcomes.

As hypothesized previously $[1,2]$, differences between AFDAS and KAF can be explained by their distinctive

Cerebrovasc Dis 2022;51:152-157 
pathophysiologies and AF screening strategies. Some AFDAS may constitute preexisting AFs diagnosed only after stroke and may bear a similar risk profile as KAF. Other AFDAS may also be preexistent but not causally associated with the stroke and incidentally diagnosed on prolonged cardiac monitoring in patients with a competing embolic (e.g., carotid artery disease) or atherothrombotic (e.g., small vessel disease) mechanisms. The Stroke of Known Cause and Underlying Atrial Fibrillation (STROKE AF) study, where AFDAS was found in $12.4 \%$ of patients with noncardioembolic events at 12 months, supports that AFDAS may not always have a causal role [12]. Regardless of the lack of a causal association between some AFDAS and the related stroke, these arrhythmias may still bear a high risk of stroke that requires close follow-up and optimized medical management. Additionally, the lower prevalence of cardiovascular comorbidities and less severe structural heart disease of AFDAS relative to KAF may be explained by stroke-related neurogenic mechanisms lowering the cardiac arrhythmogenesis threshold through autonomic dysregulation and inflammation [2]. This may occur even in individuals with apparently healthier hearts, as shown in this meta-analysis. In line with this hypothesis, 2 studies showed 3-4x higher frequency of insular strokes in AFDAS relative to $\mathrm{KAF}$, supporting a role for autonomic dysregulation [9, 10].

We hypothesize that AFDAS represents an early stage of AF relative to KAF that becomes evident either because of prolonged cardiac monitoring (e.g., low-burden AF that would remain otherwise diagnosed) or because of being triggered by neurogenic mechanisms (e.g., non-AF atrial substrate that evolves to low-burden AF because of stroke-related neurogenic mechanisms).

Considering that cardiovascular events are a common cause of death in stroke patients, we do not have a robust explanation for the lack of differences in mortality between AFDAS and KAF despite a substantially lower burden of vascular disease in the former group. We hypothesize that the risk of death in both types of AF is mainly stroke driven rather than instigated by other vascular comorbidities.

This study has limitations. Given the unusually large number of studies screened, we were unable to document the reason for the exclusion of the 33,774 studies that were not included in the analyses. We reported differences in left atrial diameter between AFDAS and KAF because this was the most consistently used measure across studies. However, other measures such as the left atrial volume index are more reliable predictors of cardio- vascular outcomes than left atrial diameter [13]. Despite this, in studies in which left atrial volume index and left atrial area were reported, sizes were consistently larger in KAF than in AFDAS patients $[10,14]$. When estimating stroke recurrence and death, we pooled unadjusted ORs (Table 1). Adjusting these results for relevant variables such as oral anticoagulation may have yielded different results. Additionally, the number of studies included in the estimate for stroke recurrence was relatively low $(n=$ 3).

\section{Conclusion}

AFDAS is possibly a different type of arrhythmia than $\mathrm{KAF}$, with a lower burden of vascular comorbidities and risk of recurrent stroke. These findings require further prospective testing.

\section{Acknowledgments}

We thank Dr. Seung-Hoon Lee and Dr. Yerim Kim for sharing unpublished data from their study.

\section{Statement of Ethics}

Given that this study only included study-level data from published sources, approval from an ethics review board or patients' consent was not required.

\section{Conflict of Interest Statement}

L.A.S. reported the following: speaker and consulting honoraria (Boehringer Ingelheim, Pfizer, Bayer, Gore); consulting honoraria (Daiichi Sankyo); Editorial Board member (Neurology, Stroke, and JAHA); Co-editor, Neurocardiology Section of Stroke; Guest Associate Editor, JAHA. Other authors have nothing to disclose.

\section{Funding Sources}

L.A.S. was supported by Kathleen \& Dr. Henry Barnett Research Chair in Stroke Research (Western University) and Edward and Alma Saraydar Neurosciences Fund (London Health Sciences Foundation). No funding source had a role in the design or reporting of the results. 


\section{Author Contributions}

S.F. contributed to study design, statistical analysis plan, statistical analysis, and manuscript drafting. A.J.-R. contributed to study design and manuscript revision for intellectual content. J.C.V.-G. contributed to study design, statistical analysis plan, and manuscript revision for intellectual content. L.A.S. contributed to study design, statistical analysis plan, study coordination, and manuscript drafting.

\section{Data Availability Statement}

Most of the data used in this study are available in the online supplementary file. Full datasets will be made available upon reasonable request.

\section{References}

1 Cerasuolo JO, Cipriano LE, Sposato LA. The complexity of atrial fibrillation newly diagnosed after ischemic stroke and tia: advances and uncertainties. Curr Opin Neurol. 2017; 30:28-37.

2 Sposato LA, Riccio PM, Hachinski V. Poststroke atrial fibrillation: cause or consequence? critical review of current views. Neurology. 2014;82:1180-6.

3 Sposato LA, Cerasuolo JO, Cipriano LE, Fang J, Fridman S, Paquet M, et al. Atrial fibrillation detected after stroke is related to a low risk of ischemic stroke recurrence. Neurology. 2018;90:e924-31.

4 Jimenez-Ruiz A, Vargas-Gonzalez JC, Fridman S, Sposato LA. Phenotypes of atrial fibrillation diagnosed before-versus-after ischaemic stroke and tia: study protocol for a systematic review and meta-analysis. BMJ open. 2021;11(7):e044288.

5 Page MJ, McKenzie JE, Bossuyt PM, Boutron I, Hoffmann TC, Mulrow CD, et al. The prisma 2020 statement: an updated guideline for reporting systematic reviews. BMJ. 2021;372:n71.

6 Higgins JPT, Thomas J, Chandler J, Cumpston M, Li T, Page MJ, et al. Cochrane handbook for systematic reviews of interventions version 6.2 (updated $2021 \mathrm{Feb}$ ). Cochrane; 2021. Available from: www.Training.Cochrane. Org/handbook. 2021.

7 Cohen J. Statistical power analysis for the behavioral sciences. 2nd ed. Hillsdale, NJ: Erlbaum; 1988.

8 Moola S, Munn Z, Tufanaru C, Aromataris E, Sears K, Sfetcu R, et al. Chapter 7: systematic reviews of etiology and risk. In: Aromataris $\mathrm{E}$, Munn Z, editors. Jbi manual for evidence synthesis. Jbi; 2020. Available from: https://synthesismanual.Jbi.

9 Vingerhoets F, Bogousslavsky J, Regli F, Van Melle G. Atrial fibrillation after acute stroke. Stroke. 1993;24:26-30.

10 Gonzalez Toledo ME, Klein FR, Riccio PM, Cassara FP, Munoz Giacomelli F, Racosta JM, et al. Atrial fibrillation detected after acute ischemic stroke: Evidence supporting the neurogenic hypothesis. J Stroke Cerebrovasc Dis. 2013;22:e486-491.

11 Sposato LA, Cipriano LE, Riccio PM, Hachinski V, Saposnik G. Very short paroxysms account for more than half of the cases of atrial fibrillation detected after stroke and tia: a sys- tematic review and meta-analysis. Int J Stroke. 2015;10:801-7.

12 Bernstein RA, Kamel H, Granger CB, Piccini JP, Sethi PP, Katz JM, et al. Effect of long-term continuous cardiac monitoring versus usual care on detection of atrial fibrillation in patients with stroke attributed to large- or smallvessel disease: the stroke-af randomized clinical trial. JAMA. 2021;325(21):2169-77.

13 Tsang TS, Abhayaratna WP, Barnes ME, Miyasaka Y, Gersh BJ, Bailey KR, et al. Prediction of cardiovascular outcomes with left atrial size: is volume superior to area or diameter? J Am Coll Cardiol. 2006;47:1018-23.

14 Baturova MA, Sheldon SH, Carlson J, Brady PA, Lin G, Rabinstein AA, et al. Electrocardiographic and echocardiographic predictors of paroxysmal atrial fibrillation detected after ischemic stroke. BMC Cardiovasc Disord. 2016;16:209.

15 Palm F, Kleemann T, Dos Santos M, Urbanek C, Buggle F, Safer A, et al. Stroke due to atrial fibrillation in a population-based stroke registry (ludwigshafen stroke study) chads(2), cha(2) ds(2) -vasc score, underuse of oral anticoagulation, and implications for preventive measures. Eur J Neurol. 2013;20:117-23.

16 Ward F, McGovern R, Cotter PE. Troponin-i is a predictor of a delayed diagnosis of atrial fibrillation in acute ischemic stroke and transient ischemic attack. J Stroke Cerebrovasc Dis. 2015;24:66-72.

17 Jaakkola J, Mustonen P, Kiviniemi T, Hartikainen JE, Palomäki A, Hartikainen P, et al. Stroke as the first manifestation of atrial fibrillation. PLoS One. 2016;11:e0168010.

18 Kim Y, Lee SH. Embolic stroke and after-admission atrial fibrillation. Int J Cardiol. 2016; 222:576-80.

19 Rizos T, Horstmann S, Dittgen F, Täger T, Jenetzky E, Heuschmann P, et al. Preexisting heart disease underlies newly diagnosed atrial fibrillation after acute ischemic stroke. Stroke. 2016;47:336-41.

20 Borowsky LH, Regan S, Chang Y, Ayres A, Greenberg SM, Singer DE. First diagnosis of atrial fibrillation at the time of stroke. Cerebrovasc Dis. 2017;43:192-9.

21 Muscari A, Bonfiglioli A, Faccioli L, Ghinelli M, Magalotti D, Manzetto F, et al. Usefulness of the mrwallets scoring system to predict first diagnosed atrial fibrillation in patients with ischemic stroke. Am J Cardiol. 2017;119: 1023-9.

22 Rizos T, Bartsch AJ, Johnson TD, Dittgen F, Nichols TE, Malzahn U, et al. Voxelwise distribution of acute ischemic stroke lesions in patients with newly diagnosed atrial fibrillation: Trigger of arrhythmia or only target of embolism? PLoS One. 2017;12:e0177474.

23 Shiroto H, Tomita H, Hagii J, Metoki N, Fujita A, Kamada T, et al. Impact of atrial natriuretic peptide value for predicting paroxysmal atrial fibrillation in ischemic stroke patients. J Stroke Cerebrovasc Dis. 2017;26:772-8.

24 Yang X, Li S, Zhao X, Liu L, Jiang Y, Li Z, et al. Atrial fibrillation is not uncommon among patients with ischemic stroke and transient ischemic stroke in china. BMC Neurol. 2017; 17:207.

25 Hsieh CY, Lee CH, Wu DP, Sung SF. Characteristics and outcomes of ischemic stroke in patients with known atrial fibrillation or atrial fibrillation diagnosed after stroke. Int J Cardiol. 2018;261:68-72.

26 Krawczyk M, Fridman S, Cheng Y, Fang J, Saposnik G, Sposato LA. Atrial fibrillation diagnosed after stroke and dementia risk: cohort study of first-ever ischaemic stroke patients aged 65 or older. Europace : European pacing, arrhythmias, and cardiac electrophysiology. Europace. 2019;21:1793-801.

27 Li SY, Yang XM, Zhao XQ, Liu LP, Wang YL, Jiang Y, et al. Newly detected atrial fibrillation is associated with cortex-involved ischemic stroke. Chin Med J. 2019;132:2053-8.

28 Yang XM, Rao ZZ, Gu HQ, Zhao XQ, Wang CJ, Liu LP, et al. Atrial fibrillation known before or detected after stroke share similar risk of ischemic stroke recurrence and death. Stroke. 2019;50:1124-9.

29 Ziegler NL, Sieweke JT, Biber S, Gabriel MM, Schuppner R, Worthmann H, et al. Markers of endothelial pathology to support detection of atrial fibrillation in embolic stroke of undetermined source. Sci Rep. 2019;9:19424.

30 Bhatla A, Borovskiy Y, Katz R, Hyman MC, Patel PJ, Arkles J, et al. Stroke, timing of atrial fibrillation diagnosis, and risk of death. Neurology. 2021;96(12):e1655-62.

31 Watanabe K, Okazaki S, Kitano T, Sugiyama S, Ohara M, Kanki H, et al. Stroke severity and outcomes in patients with newly diagnosed atrial fibrillation. Front Neurol. 2021;12:666491. 